\title{
Antimicrobial and cytotoxic activities of Alnus rugosa L. aerial parts and identification of the bioactive components
}

\author{
Khaled Rashed ${ }^{\mathrm{a}}$, Ana Ćirić ${ }^{\mathrm{b}}$, Jasmina Glamočlija ${ }^{\mathrm{b}}$, Ricardo C. Calhelha ${ }^{\mathrm{c}, \mathrm{d}}$, \\ Isabel C.F.R. Ferreirac ${ }^{c}$, Marina Soković ${ }^{b, *}$ \\ a Pharmacognosy Department, National Research Centre, Dokki, Giza, Egypt \\ b Institute for biological research “Siniša Stanković”, University of Belgrade, Blvd. despota Stefana 142, 11000 Belgrade, Serbia \\ ${ }^{\mathrm{c}}$ Mountain Research Center (CIMO), ESA, Polytechnic Institute of Bragança, Campus de Santa Apolónia, Ap. 1172, 5301-855 Bragança, Portugal \\ d Centre of Chemistry, University of Minho, Campus de Gualtar, 4710-057 Braga, Portugal
}

\section{A R T I C L E I N F O}

\section{Article history:}

Received 28 October 2013

Received in revised form 7 May 2014

Accepted 12 May 2014

\section{Keywords:}

Alnus rugosa

Antibacterial activity

Antifungal activity

Anticancer activity

Natural drugs

Phytochemicals

\begin{abstract}
A B S T R A C T
This present investigation was undertaken to study antimicrobial and cytotoxic activities of dichloromethane, ethyl acetate, and methanol 80\% extracts from Alnus rugosa L. aerial parts, as well investigate the bio-active compounds of each extract. Antimicrobial activity of the mentioned extracts against various bacteria and fungi using microdilution method, and cytotoxicity against five different human tumor cell lines, and in a non-tumor liver cells primary culture were tested. Extracts were subjected to phytochemical analysis and column chromatography eluted with different solvents, $n$-hexane, dichloromethane, ethyl acetate, and methanol gradually. The results showed that the extracts had a significant antimicrobial activity against all the tested bacteria and fungi, and proved a significant cytotoxic effect. Methanol $80 \%$ extract exhibited the highest antibacterial effect, and ethyl acetate extract was the most active as antifungal agent while dichloromethane extract showed the lowest antifungal effect. Dichloromethane extract was the most active against all the tested tumor cell lines. Chromatographic separation of dichloromethane extract of $A$. rugosa aerial parts resulted in the isolation and identification of $\beta$-sitosterol, $\beta$-sitosterol 3-O- $\beta$-glucoside and apigenin, while from ethyl acetate extract, diosmetin, naringenin, luteolin, and luteolin 7-O- $\beta$-glucoside were identified, and from methanol $80 \%$ extract, quercetin 3-O- $\alpha$-rhamnoside, isorhamnetin 3-O- $\beta$-glucoside and isorhamnetin 3-O- $\beta$-glucoside 7-O- $\alpha$-rhamnoside were identified. In conclusion, the present research provided significant information about antimicrobial and cytotoxic activities and compounds isolated from A. rugosa aerial parts extracts.
\end{abstract}

(C) 2014 Published by Elsevier B.V.

\section{Introduction}

Bacterial and fungal infections are one of the serious global health problems in 21st century (Morris and Masterton, 2002). There are several reports of antibiotic resistance of human pathogens to available antibiotics (Levy and Marshall, 2004). The multiple drug resistance and associated adverse effects of antibiotics on the host including hypersensitivity, immune suppression and allergic reaction are growing and because of this outlook the use of antimicrobial drugs in the future is still uncertain (Parekh and Chanda, 2007). There is a need of an alternative source for new

\footnotetext{
* Corresponding author. Tel.: +381 112078419.

E-mail address: mris@ibiss.bg.ac.rs (M. Soković).
}

antibiotics in the drug-development pipeline. Cancer is also one of the most life-threatening diseases and causes serious health problems in both developed and developing countries. It is a group of diseases characterized by the deregulated proliferation of abnormal cells that invade and disrupt surrounding tissues (Gennari et al., 2007). Therefore, investigations for finding new anticancer compounds are imperative and interesting. After taking into consideration the immense side effects of synthetic anticancer drugs, many researchers are making concerted efforts to find new and natural anticancer compounds. The screening of plant extracts has been of great interest to scientists in the search for new drugs for effective treatment of several diseases (Dimayuga and Garcia, 1991). Alnus rugosa plant (family Betulaceae) is a deciduous tree growing to $22 \mathrm{~m}$. It flowers in May, and the seeds ripen in October. In traditional medicine, A. rugosa is used as alterative, anodyne, astringent, cathartic, emetic, febrifuge and tonic (Huxley, 1992). 
There are no previous reports about pharmacological activities or phytochemicals from $A$. rugosa plant. The present study revealed the potential of the extracts from the mentioned plant against some gram-positive, gram-negative strains of bacteria, fungi, and evaluated the cytotoxic activity for human tumor cell lines, as well identified the bioactive components responsible for these activities in the plant extracts.

\section{Materials and methods}

\subsection{Apparatus}

Ultra violet-visible specter were done (UV/vis) by Shimadzu UV-visible recording spectrophotometer model-UV 240 (NRC, Egypt). Spectroscopic data $\left({ }^{1} \mathrm{H}-\mathrm{NMR},{ }^{13} \mathrm{C}-\mathrm{NMR}\right)$ : Varian Unity INOVA. Mass spectroscopy-MS (Finnigan MAT SSQ 7000, $70 \mathrm{eV}$ ). Silica gel (60-200 mesh, Merck) for column chromatography, thin layer chromatography (TLC): pre-coated sheets of silica gel $60 \mathrm{~F}_{254}$ (Merck). Sephadex LH-20 (Sigma).

\subsection{Plant identification and collection}

A. rugosa aerial parts were collected from Al-Zohiriya garden, Giza, Egypt in May 2011. The plant was identified by Dr. Mohammed El-Gebaly, Department of Botany, National Research Centre (NRC) and by Mrs. Tereeza Labib Consultant of Plant Taxonomy at the Ministry of Agriculture and director of Orman botanical garden, Giza, Egypt. A voucher specimen was deposited in the herbarium of Al-Zohiriya garden, Giza, Egypt.

\subsection{Preparation of plant extracts}

The air dried powder of A. rugosa aerial parts (750g) was extracted with dichloromethane, ethyl acetate and methanol:distilled water $(80: 20)(\mathrm{v} / \mathrm{v})$ by maceration method till exhaustion. The extracts were concentrated under reduced pressure to give $16 \mathrm{~g}, 7.5 \mathrm{~g}$ and $32 \mathrm{~g}$ of dichloromethane, ethyl acetate, and methanol $80 \%$ extracts, respectively. Each extract was subjected to phytochemical analysis according to different chemical assays to identify the presence or absence of the phytoconstituents according to methods described by Connolly et al. (1970) for sterols and/or triterpenes, Wolf et al. (1962) for carbohydrates and saponins, Harborne (1973) for flavonoids and alkaloids, Farnsworth (1966) for coumarins and Geissman (1962) for tannins.

\subsection{Phytochemical characterization}

Each extract was subjected to column chromatography with silica gel $(0.06-0.2 \mathrm{~mm})$ and the elution with different solvents, $n$-hexane, dichloromethane, ethyl acetate and methanol gradually.

Dichloromethane extract eluted with $n$-hexane and ethyl acetate gradually. Elution with $n$-hexane:ethyl acetate (80:20) gave compound 1, $n$-hexane:ethyl acetate (50:50) yielded compound 2 and further elution with ethyl acetate:hexane (70:30) gave compound 3.

Ethyl acetate extract eluted with dichloromethane and methanol gradually. Elution with dichloromethane:methanol $(90: 10)$ gave compound 4, dichloromethane:methanol $(80: 20)$ yielded compound 5 , elution with dichloromethane:methanol (70:30) gave compound 6 and further elution with dichloromethane:methanol (60:40) yielded compound 7.

Methanol $80 \%$ extract eluted with dichloromethane and methanol gradually: Eluction with dichloromethane:methanol 90:10 gave compound 8, dichloromethane:methanol $(80: 20)$ yielded compound 9, and further elution with methanol:dichloromethane (60:40) gave compound 10. All the isolated compounds were purified over sephdex LH-20 column chromatography eluted with methanol and methanol/water mixture.

\subsection{Acid hydrolysis of flavonoid glycosides}

Solutions of $5 \mathrm{mg}$ of compounds 7, 8, 9, and 10 in $5 \mathrm{~mL}$ of $10 \%$ $\mathrm{HCl}$ was heated for $5 \mathrm{~h}$. The aglycones were extracted with EtOAc and identified by co-TLC with authentic standards. The sugars in the aqueous layer was identified by co-paper chromatography (coPC) with authentic markers on Whatman No. 1 sheets in solvent system (n-BuOH-AcOH- $\mathrm{H}_{2} \mathrm{O}$ 4:1:5 upper layer).

\subsection{Antibacterial activity}

The following Gram (-) bacteria, Enterobacter cloacae human isolate, Escherichia coli (ATCC 35210), Pseudomonas aeruginosa (ATCC 27853), and Salmonella typhimurium (ATCC 13311) and Gram (+) bacteria, Bacillus cereus (clinical isolate), Listeria monocytogenes (NCTC 7973), Micrococcus flavus (ATCC 10240), and Staphylococcus aureus (ATCC 6538) were used. The organisms were obtained from Mycological Laboratory, Department of Plant Physiology, Institute for Biological Research "Siniša Stanković", University of Belgrade, Serbia. The antibacterial assay was carried out by microdilution method (CLSI, 2009; Tsukatani et al., 2012). The bacterial suspensions were adjusted with sterile saline to a concentration of $1.0 \times 10^{5} \mathrm{CFU} / \mathrm{mL}$. The inocula were prepared daily and stored at $4{ }^{\circ} \mathrm{C}$ until use. Dilutions of the inocula were cultured on solid medium to verify the absence of contamination and to check the validity of the inoculum. The minimum inhibitory and bactericidal concentrations (MICs and MBCs) were determined using 96-well microtitre plates. The bacterial suspension was adjusted with sterile saline to a concentration of $1.0 \times 10^{5} \mathrm{CFU} / \mathrm{mL}$. The tested extracts were added ( 1 and $10 \mathrm{mg} / \mathrm{mL}$ ) in broth Triptic Soy broth (TSB) medium $(100 \mu \mathrm{L})$ with bacterial inoculum $\left(1.0 \times 10^{4} \mathrm{CFU}\right.$ per well $)$ to achieve the wanted concentrations. The microplates were incubated at rotary shaker $(160 \mathrm{rpm})$ for $24 \mathrm{~h}$ at $37^{\circ} \mathrm{C}$. The following day, $30 \mu \mathrm{L}$ of $0.2 \mathrm{mg} / \mathrm{mL}$ solution of INT ( $p$-iodonitrotetrazolium violet) was added, and the plates were returned to the incubator for at least one-half hour to ensure adequate color reaction. Inhibition of growth was indicated by a clear solution or a definite decrease in color reaction. The lowest concentrations without visible growth (at the binocular microscope) were defined as concentrations that completely inhibited bacterial growth (MICs). The MBCs were determined by serial sub-cultivation of $2 \mu \mathrm{L}$ into microtitre plates containing $100 \mu \mathrm{L}$ of broth per well and further incubation for $24 \mathrm{~h}$. The lowest concentration with no visible growth was defined as the MBC, indicating 99.5\% killing of the original inoculum. The optical density of each well was measured at a wavelength of $655 \mathrm{~nm}$ by microplate manager 4.0 (Bio-Rad Laboratories) and compared with a blank and the positive control. The antibiotics streptomycin and ampicillin were used as positive controls $(1 \mathrm{mg} / \mathrm{mL}$ in sterile physiological saline). Three independent experiments were performed in triplicate.

\subsection{Antifungal activity}

The used fungi: Aspergillus fumigatus (ATCC 1022), Aspergillus versicolor (ATCC 11730), Aspergillus ochraceus (ATCC 12066), Aspergillus niger (ATCC 6275), Trichoderma viride (IAM 5061), Penicillium funiculosum (ATCC 36839), Penicillium ochrochloron (ATCC 9112), and Penicillium verrucosum var. cyclopium were obtained from Mycological Laboratory, Department of Plant Physiology, Institute for Biological Research "Siniša Stanković", University of Belgrade, Serbia. 
The micromycetes were maintained on malt agar and the cultures were stored at $4{ }^{\circ} \mathrm{C}$ and sub-cultured once a month. The antifungal assay was carried out by modified microdilution technique (Hanel and Raether, 1988; Espinel-Ingroff, 2001). The fungal spores were washed from the surface of agar plates with sterile $0.85 \%$ saline containing $0.1 \%$ Tween $80(\mathrm{v} / \mathrm{v})$. The spore suspension was adjusted with sterile saline to a concentration of approximately $1.0 \times 10^{5}$ in a final volume of $100 \mu \mathrm{L}$ per well. The inocula were stored at $4{ }^{\circ} \mathrm{C}$ for further use. Dilutions of the inoculum were cultured on solid malt agar to verify the absence of contamination and to check the validity of the inoculum. Minimal inhibitory concentration (MIC) determinations were performed by a serial dilution technique using 96-well microtiter plates. The examined extracts were diluted in $5 \%$ of DMSO $(1 \mathrm{mg} / \mathrm{mL}$ and $10 \mathrm{mg} / \mathrm{mL})$ and added in broth Malt medium (MA) with inoculum. The microplates were incubated at rotary shaker $(160 \mathrm{rpm})$ for $72 \mathrm{~h}$ at $28^{\circ} \mathrm{C}$. The lowest concentrations without visible growth (at the binocular microscope) were defined as MICs. The fungicidal concentrations (MFCs) were determined by serial subcultivation of $2 \mu \mathrm{L}$ of tested fractions dissolved in medium and inoculated for $72 \mathrm{~h}$, into microtiter plates containing $100 \mu \mathrm{L}$ of broth per well and further incubation $72 \mathrm{~h}$ at $28^{\circ} \mathrm{C}$. The lowest concentration with no visible growth was defined as MFC indicating 99.5\% killing of the original inoculum. The fungicides bifonazole and ketoconazole were used as positive controls (1-3500 $\mu \mathrm{g} / \mathrm{mL})$. Three independent experiments were performed in duplicate.

\subsection{Cytotoxicity in human tumor cell lines and in liver cells primary cell culture}

Five human tumor cell lines were used: MCF-7 (breast adenocarcinoma), NCI-H460 (non-small cell lung cancer), HCT-15 (colon carcinoma), HeLa (cervical carcinoma) and HepG2 (hepatocellular carcinoma). Cells were routinely maintained as adherent cell cultures in RPMI-1640 medium containing 10\% heat-inactivated fetal bovine serum (FBS) and $2 \mathrm{mM}$ glutamine (MCF-7, NCI-H460 and HCT-15) or in DMEM supplemented with 10\% FBS, 2 mM glutamine, $100 \mathrm{U} / \mathrm{mL}$ penicillin and $100 \mathrm{mg} / \mathrm{mL}$ streptomycin (HeLa and HepG2 cells), at $37^{\circ} \mathrm{C}$, in a humidified air incubator containing $5 \% \mathrm{CO}_{2}$. Each cell line was plated at an appropriate density ( 7.5 $\times 10^{3}$ cells/well for MCF-7, NCI-H460 and HCT-15 or $1.0 \times 10^{4}$ cells/well for HeLa and HepG2) in 96-well plates. Sulforhodamine B assay was performed according to a procedure previously described by Guimarães et al. (2013). For hepatotoxicity evaluation, a cell culture was prepared from a freshly harvested porcine liver obtained from a local slaughter house, according to a procedure established by Guimarães et al. (2013); it was designed as PLP2. Cultivation of the cells was continued with direct monitoring every two to three days using a phase contrast microscope. Before confluence was reached, cells were subcultured and plated in 96-well plates at a density of $1.0 \times 10^{4}$ cells/well, and cultivated in DMEM medium with $10 \% \mathrm{FBS}, 100 \mathrm{U} / \mathrm{mL}$ penicillin and $100 \mu \mathrm{g} / \mathrm{mL}$ streptomycin. Three independent experiments were performed in triplicate.

\section{Results}

\subsection{Phytochemical characterization of A. rugosa aerial parts extracts}

Phytochemical analysis revealed that dichloromethane extract had triterpenes and/or sterols, and flavonoids. Ethyl acetate extract contained carbohydrates, triterpenes and/or sterols, and flavonoids while methanol $80 \%$ extract had carbohydrates, triterpenes and/or sterols, flavonoids and tannins (Table 1). The major bioactive components identified from $A$. rugosa dichloromethane extract were $\beta$-sitosterol, $\beta$-sitosterol 3 - $O$ - $\beta$-glucoside, and apigenin, while in ethyl acetate extract, diosmetin, narigenin, luteolin, and luteolin 7-O- $\beta$-glucoside were the most abundant. Quercetin3-O$\alpha$-rhamnoside, isorhamnetin 3-O- $\beta$-glucoside, and isorhamnetin $3-O$ - $\beta$-glucoside $7-O-\alpha$-rhamnoside were identified in methanol $80 \%$ extract (Fig. 1). The chemical structures of the mentioned compounds were elucidated by different spectroscopic analyses (UV, ${ }^{1} \mathrm{H}-\mathrm{NMR},{ }^{13} \mathrm{C}-\mathrm{NMR}$ and MS).

\subsection{Structure elucidation of the isolated compounds of A. rugosa} extracts

Compound 1 ( $\beta$-sitosterol) was isolated as white needles, $16 \mathrm{mg}$. ${ }^{1} \mathrm{H}-\mathrm{NMR}\left(\mathrm{CDCl}_{3}, 400 \mathrm{MHz}\right.$ ) : $\delta 5.37(1 \mathrm{H}, \mathrm{m}, \mathrm{H}-6), 3.52(1 \mathrm{H}, \mathrm{m}, \mathrm{H}-3)$, 1.09 (3H, s, $\left.\mathrm{CH}_{3}-19\right), 0.98$ (3H, d, $\left.J=6.5, \mathrm{CH}_{3}-21\right), 0.92(3 \mathrm{H}, \mathrm{t}, J=7.4$, $\left.\mathrm{CH}_{3}-29\right), 0.85\left(3 \mathrm{H}, \mathrm{d}, \mathrm{J}=6.7 \mathrm{~Hz}, \mathrm{CH}_{3}-26\right), 0.81\left(3 \mathrm{H}, \mathrm{d}, \mathrm{J}=6.7 \mathrm{~Hz}, \mathrm{CH}_{3}-\right.$ 27), $0.75\left(3 \mathrm{H}, \mathrm{s}, \mathrm{CH}_{3}-18\right) .{ }^{13} \mathrm{C}-\mathrm{NMR}\left(\mathrm{CDCl}_{3}, 100 \mathrm{MHz}\right): \delta 140.48(\mathrm{C}-5)$, 121.54 (C-6), 71.62 (C-3), 57.28 (C-17), 56.42 (C-14), 50.34 (C-9), 46.38 (C-24), 42,82 (C-13, 4), 39.86 (C-12), 37.64 (C-1), 36.76 (C10), 35.92 (C-20), 34.28 (C-22), 31.74 (C-8, 7), 31.42 (C-2), 29.28 (C-25), 28.44 (C-16), 26.24 (C-23), 24.54 (C-15), 23.42 (C-28), 21.18 (C-11), 19.82 (C-26), 19.56 (C-19), 19.26 (C-27), 18.64 (C-21).

Compound 2 ( $\beta$-sitosterol 3-O- $\beta$-glucoside) was isolated as white crystals, $12 \mathrm{mg} .{ }^{1} \mathrm{H}-\mathrm{NMR}\left(\mathrm{CDCl}_{3}, 400 \mathrm{MHz}\right): \delta 1.27(2 \mathrm{H}, \mathrm{m}$, $\mathrm{H}-1), 1.35$ (2H, m, H-2, 11, 12, 28), $3.13(1 \mathrm{H}, \mathrm{m}, \mathrm{H}-3), 2.16$ (2H, m, $\mathrm{H}-4), 5.12$ (1H, m, H-6), 1.76 ( $2 \mathrm{H}, \mathrm{m}, \mathrm{H}-7, \mathrm{H}-15,16,17,22,23), 1.24$ (1H, m, H-8, 9, 14), 0.64 (3H, s, H-18), 0.96 (3H, s, H-19), 1.36 (1H, $\mathrm{m}, \mathrm{H}-20), 0.82(3 \mathrm{H}, \mathrm{d}, \mathrm{J}=6.2 \mathrm{~Hz}, \mathrm{H}-21), 1.14(1 \mathrm{H}, \mathrm{m}, \mathrm{H}-24), 2.17(1 \mathrm{H}$, $\mathrm{m}, \mathrm{H}-25) 0.78$ (3H, d, J= 7.5 Hz, H-26), 0.75 (3H, d, $J=1.7 \mathrm{~Hz}, \mathrm{H}-27)$, $0.76(3 \mathrm{H}, \mathrm{t}, J=6.8 \mathrm{~Hz}, \mathrm{H}-29), 4.12\left(1 \mathrm{H} \mathrm{d}, J=7.7 \mathrm{~Hz}, \mathrm{H}-1^{\prime}\right), 3.12(1 \mathrm{H}, \mathrm{m}$, H-2' $\left.3^{\prime}, 4^{\prime}\right), 3.07\left(1 H, \mathrm{~m}, \mathrm{H}-5^{\prime}\right), 2.97\left(1 \mathrm{H}, \mathrm{m}, \mathrm{H}-6^{\prime}\right)$. EI/MS: $m / z 576$.

Compound 3 (apigenin) was isolated as a yellow powder, $20 \mathrm{mg}$. ${ }^{1} \mathrm{H}-\mathrm{NMR}\left(\mathrm{CD}_{3} \mathrm{OD}, 500 \mathrm{MHz}\right): \delta 7.85\left(2 \mathrm{H}, \mathrm{d}, J=8.5 \mathrm{~Hz}, \mathrm{H}-2^{\prime}, \mathrm{H}^{\prime}\right), 6.95$ $\left(2 \mathrm{H}, \mathrm{d}, J=8.5 \mathrm{~Hz}, \mathrm{H}^{-} 3^{\prime}, \mathrm{H}^{-} 5^{\prime}\right), 6.54(1 \mathrm{H}, \mathrm{s}, \mathrm{H}-3), 6.42(1 \mathrm{H}, \mathrm{s}, \mathrm{H}-8), 6.28$ (1H, s, H-6). EI-MS: $m / z 270$.

Compound 4 (diosmetin) was isolated as a yellow powder, $14 \mathrm{mg}$. UV $\lambda_{\max }(\mathrm{MeOH}): 247,264,345$, (NaOMe): 249, 302, 388, $\left(\mathrm{AlCl}_{3}\right): 273,352,400\left(\mathrm{AlCl}_{3} / \mathrm{HCl}\right): 272,352,400$, (NaOAc): 252, 382, $\left(\mathrm{NaOAc} / \mathrm{H}_{3} \mathrm{BO}_{3}\right): 252,382 .{ }^{1} \mathrm{H}$ NMR $\left(\mathrm{DMSO}^{-} \mathrm{d}_{6}, 500 \mathrm{MHz}\right): \delta 7.65$ $\left(1 \mathrm{H}, \mathrm{d}, J=2.4 \mathrm{~Hz}, \mathrm{H} 2^{\prime}\right) ; \delta$ ppm $7.35\left(1 \mathrm{H}, \mathrm{dd}, J=7.6,2.4 \mathrm{~Hz}, \mathrm{H}-6^{\prime}\right) ; 6.85$ $\left(1 \mathrm{H}, \mathrm{d}, J=7.6 \mathrm{~Hz}, \mathrm{H}-5^{\prime}\right) ; 6.68(1 \mathrm{H}, \mathrm{d}, J=2.4 \mathrm{~Hz}, \mathrm{H}-8) ; 6.45(1 \mathrm{H}, \mathrm{d}, J=2$. $2 \mathrm{~Hz}, \mathrm{H6}$ ); 6.65 (1H, s, H3); $3.92\left(3 \mathrm{H}, \mathrm{s}, \mathrm{OCH}_{3}\right) .{ }^{13} \mathrm{C}-\mathrm{NMR}$ (DMSO-d 6 , $100 \mathrm{MHz}$ ): $\delta$ ppm 162.94 (C-2), 103.86 (C-3), 182.62 (C-4), 161.48 (C-5), 98.76 (C-6), 164.74 (C-7), 94.28 (C-8), 157.64 (C-9), 104.26 (C$\left.10), 123.48\left(\mathrm{C}-1^{\prime}\right)\right), 112.88\left(\mathrm{C}-2^{\prime}\right), 147.34\left(\mathrm{C}-3^{\prime}\right), 151.42\left(\mathrm{C}-4^{\prime}\right), 112.75$ $\left(\mathrm{C}-5^{\prime}\right), 119.16\left(\mathrm{C}-6^{\prime}\right), 55.64\left(\mathrm{OCH}_{3}\right)$.

Compound 5 (naringenin) was isolated as a yellow needles, $18 \mathrm{mg}$. UV $\lambda_{\max }(\mathrm{nm}):(\mathrm{MeOH}): 289,324 \mathrm{sh}$, (NaOMe): 246, 265sh, 328, $\left(\mathrm{AlCl}_{3}\right): 312,374\left(\mathrm{AlCl}_{3} / \mathrm{HCl}\right)$ 312, 375, (NaOAc): 294sh, 325 , $\left(\mathrm{NaOAc} / \mathrm{H}_{3} \mathrm{BO}_{3}\right): 290,324 \mathrm{sh}, 332 \mathrm{sh} .{ }^{1} \mathrm{H}-\mathrm{NMR}(\mathrm{CD} 3 \mathrm{OD}, 300 \mathrm{MHz}$, ppm $2.95(1 \mathrm{H}, \mathrm{dd}, J=1.7,9.5 \mathrm{~Hz}, \mathrm{H}-3 \alpha), 3.02(1 \mathrm{H}, \mathrm{dd}, J=9.5,12.4 \mathrm{~Hz}$, H-3ß), 5.15 ( $1 H$, dd, $J=1.7,9.5 \mathrm{~Hz}, \mathrm{H}-2 \alpha), 5.78$ (2H, s, H-6, 8), 6.72 $\left(2 \mathrm{H}, \mathrm{d}, J=6.5 \mathrm{~Hz}, \mathrm{H}-3^{\prime}, 5^{\prime}\right), 7.28\left(2 \mathrm{H}, \mathrm{d}, J=6.5 \mathrm{~Hz}, \mathrm{H}-2^{\prime}, 6^{\prime}\right)$. EI-MS: $m / z$ 272.

Compound 6 (luteolin) was isolated as a yellow powder, $15 \mathrm{mg}$. ${ }^{1} \mathrm{H}-\mathrm{NMR}\left(\mathrm{CD}_{3} \mathrm{OD}, 500 \mathrm{MHz}\right): \delta \mathrm{ppm} 12.98(1 \mathrm{H}, \mathrm{s}, 5-\mathrm{OH}), 7.42(1 \mathrm{H}, \mathrm{d}$, $\left.J=8 \mathrm{~Hz}, \mathrm{H}-6^{\prime}\right), 7.38\left(1 \mathrm{H}, \mathrm{d}, J=2 \mathrm{~Hz}, \mathrm{H}-2^{\prime}\right), 6.85\left(1 \mathrm{H}, \mathrm{d}, J=8 \mathrm{~Hz}, \mathrm{H}-5^{\prime}\right)$, 6.64 (1H s, H-3), 6.42 (1H, d, J = 2 Hz, H-8), 6.15 (1H, d, J=2 Hz, H-6). EI-MS: $m / z 286$.

Compound 7 (luteolin 7-O- $\beta$-glucoside) was isolated as yellow crystals, $22 \mathrm{mg} .{ }^{1} \mathrm{H}-\mathrm{NMR}\left(\mathrm{CD}_{3} \mathrm{OD}, 500 \mathrm{MHz}\right.$, ): $\delta 7.45(1 \mathrm{H}, \mathrm{d}, J=2.4 \mathrm{~Hz}$, $\left.\mathrm{H}-2^{\prime}\right), 7.42\left(1 \mathrm{H}, \mathrm{dd}, J=2.5,8.5 \mathrm{~Hz}, \mathrm{H}-6^{\prime}\right), 6.85(1 \mathrm{H}, \mathrm{d}, J=8.5 \mathrm{~Hz}, \mathrm{H}-$ $\left.5^{\prime}\right), 6.75(1 \mathrm{H}, \mathrm{d}, J=2.5 \mathrm{~Hz}, \mathrm{H}-8), 6.55(1 \mathrm{H}, \mathrm{s}, \mathrm{H}-3), 6.48(1 \mathrm{H}, \mathrm{d}$, $J=2.5 \mathrm{~Hz}, \mathrm{H}-6), 5.28\left(1 \mathrm{H}, \mathrm{d}, J=8.2 \mathrm{~Hz}, \mathrm{H}-1^{\prime \prime}\right), 3.9-3.2$ (rest of sugar protons, $\mathrm{H}-2^{\prime \prime}$ to $\left.\mathrm{H}-6^{\prime \prime}\right) .{ }^{13} \mathrm{C}-\mathrm{NMR}\left(\mathrm{CD}_{3} \mathrm{OD}, 100 \mathrm{MHz}\right.$,): $\delta 164.82(\mathrm{C}-$ 2), 102.96 (C-3), 182.54 (C-4), 161.48 (C-5), 99.22 (C-6), 163.45 
Table 1

Phytochemical analysis of the extracts from Alnus rugosa aerial parts.

\begin{tabular}{|c|c|c|c|}
\hline Chemical Constituents & Dichloromethane & Ethyl acetate & Methanol 80\% \\
\hline Carbohydrates and/or glycosides & - & + & + \\
\hline \multicolumn{4}{|l|}{ Tannins } \\
\hline a. Condensed tannins & - & - & + \\
\hline b. Hydrolysable tannins & - & - & + \\
\hline Alkaloids and/or nitrogenous bases & - & - & - \\
\hline Flavonoids & + & + & + \\
\hline Sterols and/or triterpenes & + & + & + \\
\hline Saponins & - & - & - \\
\hline Coumarins & - & - & - \\
\hline
\end{tabular}

$(+)$ Denotes the presence of the constituents, (-) denotes the absence of the constituents.

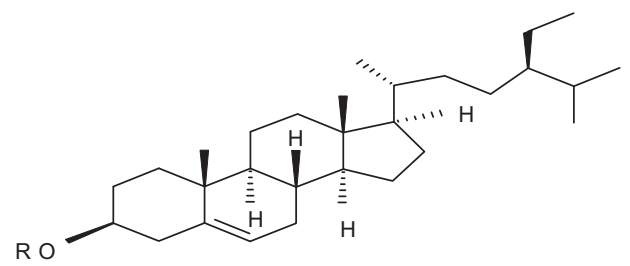

B-Sitosterol $(\mathrm{R}=\mathrm{H})$ 1:

2: B-Sitosterol 3-O-B-glucoside ( $\mathrm{R}=$ glucose)<smiles>[R]c1ccc(-c2cc(=O)c3c(O)cc(O)cc3o2)cc1[R]</smiles><smiles>O=C1CC(c2ccc(O)cc2)Oc2cc(O)cc(O)c21</smiles>

3: Apigenin $(\mathrm{R}=\mathrm{H}, \mathrm{R} 1=\mathrm{OH})$

5: Naringenin

4: Diosmetin $\left(\mathrm{R}=\mathrm{OH}, \mathrm{R} 1=\mathrm{OCH}_{3}\right)$<smiles>[R]Oc1cc(O)c2c(=O)cc(-c3ccc(O)c(O)c3)oc2c1</smiles>

6: Luteolin $(\mathrm{R}=\mathrm{H})$

7: Luteolin 7-O- $\beta$-glucside $(\mathrm{R}=$ glucose $)$<smiles>[R]Oc1cc(O)c2c(=O)c(O[R2])c(-c3ccc(O)c([R2])c3)oc2c1</smiles>

8: Quercetin 3-O- $\alpha$-rhamnoside $(\mathrm{R}=\mathrm{H}, \mathrm{R} 1=$ rhmanose, $\mathrm{R} 2=\mathrm{OH})$

9: Isorhamnetin 3-O- $\beta$-glucoside $\left(\mathrm{R}=\mathrm{H}, \mathrm{R} 1=\right.$ glucose, $\left.\mathrm{R} 2=\mathrm{OCH}_{3}\right)$

10: Isorhamnetin 3- $\beta$-O-glucoside-7-O- $\alpha$-rhamnoside $\left(\mathrm{R}=\right.$ rhamnose, $\mathrm{R} 1=$ glucose, $\left.\mathrm{R} 2=\mathrm{OCH}_{3}\right)$

Fig. 1. Chemical structures of the isolated compounds from A. rugosa aerial parts extracts.

(C-7), 94.78 (C-8), 156.82 (C-9), $105.64(\mathrm{C}-10), 122.42\left(\mathrm{C}-1^{\prime}\right), 114.15$ (C-2'), 145.97 (C-3'), 150.25 (C-4'), 116.48 (C-5'), 119.72 (C-6'), $100.28\left(\mathrm{C}-1^{\prime \prime}\right), 73.46\left(\mathrm{C}-2^{\prime \prime}\right), 76.75\left(\mathrm{C}-3^{\prime \prime}\right), 69.35\left(\mathrm{C}-4^{\prime \prime}\right), 77.84\left(\mathrm{C}-5^{\prime \prime}\right)$, $60.82\left(\mathrm{C}-6^{\prime \prime}\right)$.
Compound 8 (quercetin 3-O- $\alpha$-rhamnoside) was isolated as yellow crystals, 18 mg. ${ }^{1} \mathrm{H}-\mathrm{NMR}$ (DMSO-d 6 , $\left.400 \mathrm{MHz}\right) \delta \mathrm{ppm} 7.26(2 \mathrm{H}$ m, H-2', 6'), $6.83\left(1 \mathrm{H}, \mathrm{d}, J=9 \mathrm{~Hz}, \mathrm{H}-5^{\prime}\right), 6.49(1 \mathrm{H}, \mathrm{d}, J=2.5 \mathrm{~Hz}, \mathrm{H}-8)$ $6.14(1 \mathrm{H}, \mathrm{d}, J=2.5 \mathrm{~Hz}, \mathrm{H}-6), 5.25\left(1 \mathrm{H}, \mathrm{s}, \mathrm{H}-1^{\prime \prime}\right) 0.78(3 \mathrm{H}, \mathrm{d}, J=6 \mathrm{~Hz})$. 
${ }^{13} \mathrm{C}-\mathrm{NMR}$ (DMSO-d $6,100 \mathrm{MHz}$ ): $\delta$ ppm 177.42 (C-4), 167.45 (C-7), 161.42 (C-5), 157.01 (C-2), 157.58 (C-9), 149.19 (C-4'), 145.57 (C$\left.3^{\prime}\right), 134.12$ (C-3), $131.97\left(\mathrm{C}-6^{\prime}\right), 121.48\left(\mathrm{C}-1^{\prime}\right), 115.71\left(\mathrm{C}-2^{\prime}\right), 115.42$ (C-5'), 103.18 (C-10), 101.97 (C-1"), 99.98 (C-6), 94.47 (C-8), 71.47 (C-4"), 70.94 (C-2" ), 70.85 (C-5"), $70.62\left(\mathrm{C}-3^{\prime \prime}\right), 17.78$ (C6").

Compound 9 (isorhamnetin $3-O-\beta$-glucoside) was obtained as yellow needles, $28 \mathrm{mg} .{ }^{1} \mathrm{H}-\mathrm{NMR}\left(\mathrm{CD}_{3} \mathrm{OD}, 500 \mathrm{MHz}\right): \delta$ ppm 7.85 $\left(1 \mathrm{H}, \mathrm{s}, \mathrm{H}-2^{\prime}\right), 7.54\left(1 \mathrm{H}, \mathrm{d}, J=8 \mathrm{~Hz}, \mathrm{H}^{\prime}\right), 6.95\left(1 \mathrm{H}, \mathrm{d}, J=8 \mathrm{~Hz}, \mathrm{H}-5^{\prime}\right)$, 6.45 (1H, s, H-8), 6.25 (1H, s, H-6), $5.35\left(1 \mathrm{H}, \mathrm{d}, J=6.5 \mathrm{~Hz}, \mathrm{H}-1^{\prime \prime}\right)$, $3.85\left(3 \mathrm{H}, \mathrm{s}, \mathrm{OCH}_{3}\right), 3.9-3.2$ (rest of sugar protons, $\mathrm{H}-2^{\prime \prime}$ to $\left.\mathrm{H}-6^{\prime \prime}\right)$. ${ }^{13} \mathrm{C}-\mathrm{NMR}\left(\mathrm{CD}_{3} \mathrm{OD}, 100 \mathrm{MHz}\right): \delta 157.94(\mathrm{C}-2), 134.86$ (C-3), 178.94 (C-4), 162.96 (C-5), 100.28 (C-6), 165.82 (C-7), 94.88 (C-8), 157.82 (C-9), 106.28 (C-10), 122.75 (C-1'), $116.83\left(\mathrm{C}-2^{\prime}\right), 151.47\left(\mathrm{C}-3^{\prime}\right)$, $147.96\left(\mathrm{C}-4^{\prime}\right), 115.25\left(\mathrm{C}-5^{\prime}\right), 124.32\left(\mathrm{C}-6^{\prime}\right), 104.38\left(\mathrm{C}-1^{\prime \prime}\right), 76.75$ (C$\left.2^{\prime \prime}\right), 78.28\left(\mathrm{C}-3^{\prime \prime}\right), 71.54\left(\mathrm{C}-4^{\prime \prime}\right), 78.93\left(\mathrm{C}-5^{\prime \prime}\right), 62.55\left(\mathrm{C}-6^{\prime \prime}\right), 56.65$ $\left(\mathrm{O}-\mathrm{CH}_{3}\right)$. EI-MS: $m / z 316$.

Compound 10 (isorhamnetin 3- $\beta$-O-glucoside-7- $O-\alpha$ rhamnoside) was obtained as yellow crystals, $25 \mathrm{mg}$. UV $\lambda_{\max }$ (nm): (MeOH): 261, 354, (NaOMe): 262, 404, $\left(\mathrm{AlCl}_{3}\right): 257,295$, 352, $402\left(\mathrm{AlCl}_{3} / \mathrm{HCl}\right): 258$ 295, 248 402, (NaOAc): 262, 349, $\left(\mathrm{NaOAc} / \mathrm{H}_{3} \mathrm{BO}_{3}\right): 262,350,405 .{ }^{1} \mathrm{H}-\mathrm{NMR}\left(\mathrm{DMSO}-\mathrm{d}_{6}, 300 \mathrm{MHz}\right.$ ) : $\delta$ ppm $7.85\left(1 \mathrm{H}, \mathrm{d}, J=1.9 \mathrm{~Hz}, \mathrm{H}-2^{\prime}\right), \delta 7.65(1 \mathrm{H}, \mathrm{dd}, J=8.4 \mathrm{~Hz}, 1.9 \mathrm{~Hz}$, H-6' $6^{\prime}, \delta 6.82\left(1 H, \mathrm{~d}, J=8.4 \mathrm{~Hz}, \mathrm{H}-5^{\prime}\right), 6.72(1 \mathrm{H}, \mathrm{d}, J=1.9 \mathrm{~Hz}, \mathrm{H}-8)$, 6.45 ( $1 \mathrm{H}, \mathrm{d}, J=1.9 \mathrm{~Hz}, \mathrm{H}-6), 5.42\left(1 \mathrm{H}, \mathrm{d}, J=6.9 \mathrm{~Hz} \mathrm{H}-1^{\prime \prime}\right), 5.45(1 \mathrm{H}$, d, $\left.J=1.9 \mathrm{~Hz}, \mathrm{H}-1^{\prime \prime \prime}\right), 3.75\left(3 \mathrm{H}, \mathrm{s}, \mathrm{OCH}_{3}\right), 1.12(3 \mathrm{H}, \mathrm{d}, J=6.0 \mathrm{~Hz}$, $\mathrm{CH}_{3}$ of rhamnose sugar), $3.1-4\left(9 \mathrm{H}, \mathrm{m}, \mathrm{H}-2^{\prime \prime}\right.$ : to $\mathrm{H}-6^{\prime \prime}, \mathrm{H}-2^{\prime \prime \prime}$ to $\left.\mathrm{H}-5^{\prime \prime \prime}\right)$.

\subsection{Identification of the bio-active compounds isolated from A. rugosa extracts}

Chromatographic separation and purification of dichloromethane extract resulted in the isolation and identification of compound 1 ( $\beta$-sitosterol) which gave a dark spot under short UV light and also gave a violet color after spraying with vanillin sulfuric and heating in an oven at $110^{\circ} \mathrm{C}$ for $5 \mathrm{~min}$ and compound 2 ( $\beta$-sitosterol 3-O-glucoside) which gave grey color after spraying with vanillin sulfuric and heating in an oven at $110^{\circ} \mathrm{C}$ for $5 \mathrm{~min}$. NMR spectral signals have shown signals very close to $\beta$-sitosterol) and ( $\beta$-sitosterol 3 - $O$ - $\beta$-glucoside) (Pateh et al., 2009; Yinhong et al., 2009) and confirmation of the two compounds was done by co-TLC with authentic standards. Compound 3 (apigenin) yielded a deep purple spot under UV light and changed to yellow when subjected to ammonia and $\mathrm{AlCl}_{3}$ and its spectral data was very similar to apigenin (Ersoz et al., 2002).

Chromatographic separation and purification of the ethyl acetate extract resulted in the isolation and identification of compound 4 (diosmetin) which gave deep purple spot under UV light and changed to yellow when subjected to ammonia and $\mathrm{AlCl}_{3}$ and its spectral data was in accordance with reported by Saeidnia et al. (2011). Compound 5 (naringenin) was obtain as a deep purple spot under UV light and changed to yellow when subjected to ammonia and $\mathrm{AlCl}_{3}$. Spectral data was very close to that described by AbdelRahim et al. (2003). Compound 6 (luteolin) was obtained as a deep purple spot under UV light and changed to yellow when subjected to ammonia and $\mathrm{AlCl}_{3}$ and spectral data was in accordance with reported by Saeidnia et al. (2011). Compound 7 (luteolin 7-O- $\beta$ glucoside) gave a deep purple spot under UV light and changed to yellow when subjected to ammonia and $\mathrm{AlCl}_{3}$. Acid hydrolysis yielded luteolin as an aglycone and glucose as sugar moiety and its spectral data was in accordance with that described by Saxena and Goutam (2008).

Chromatographic separation and purification of the methanol $80 \%$ extract resulted in the isolation and identification of compound 8 (quercetin 3-O- $\alpha$-rhamnoside) which gave a deep purple spot under UV light and changed to yellow when subjected to ammonia and $\mathrm{AlCl}_{3}$. Acid hydrolysis yielded quercetin as an aglycone and rhamnose as sugar moiety and its spectral data was very similar to that described by Soliman et al. (2002). Compound 9 (isorhamnetin 3-O- $\beta$-glucoside) was obtained as a deep purple spot under UV light and changed to yellow when subjected to ammonia and $\mathrm{AlCl}_{3}$. Acid hydrolysis afforded isorhamnetin as an aglycone and glucose as sugar moiety and spectral data was very similar to that described by Compound 10 (isorhamnetin 3- $\beta$ - $O$-glucoside $7-O-\alpha$ rhamnoside) was obtained as a deep brown spot changed to lemon yellow when exposed to ammonia vapor and gave yellow color with $\mathrm{AlCl}_{3}$ reagent. Complete acid hydrolysis of compound 10 revealed the presence of isorhamnetin as an aglycone and glucose and rhamnose as sugar moieties, and its spectral data was very close to that described by Wang et al. (2012).

\subsection{Antimicrobial activities}

The present investigation evaluated the antimicrobial activity and cytotoxic effect of the extracts from A. rugosa aerial parts. The results of antibacterial activity of dichloromethane, ethyl acetate and methanol 80\% extracts from $A$. rugosa are presented in Table 2. Inhibitory activity of these extracts was achieved at concentration of $0.3-2.0,0.3-2.5$, and $0.3-1.25 \mathrm{mg} / \mathrm{mL}$, while bactericidal effect was obtained at $0.4-4.0,0.4-5.0$, and $0.5-2.5 \mathrm{mg} / \mathrm{mL}$, respectively. The most sensitive bacterial species was $B$. cereus, while L. monocytogenes was the most resistant species to the tested extracts. Nevertheless, in general, both antibiotics showed better activity than the tested extracts. Streptomycin possessed MICs of $0.05-0.25 \mathrm{mg} / \mathrm{mL}$ and MBCs of $0.10-0.50 \mathrm{mg} / \mathrm{mL}$, while ampicillin revealed MICs of $0.10-0.30 \mathrm{mg} / \mathrm{mL}$ and MBCs of $0.15-0.50 \mathrm{mg} / \mathrm{mL}$. Methanol 80\% extract showed the highest antibacterial activity and ethyl acetate showed the lowest effect on tested bacteria.

Antifungal potential of the tested extracts of $A$. rugosa is presented in Table 3. The extract inhibited all microfungi at $0.3-2.5$, $0.075-0.3$ and $0.15-1.25 \mathrm{mg} / \mathrm{mL}$ (MIC) and completely stopped the growth (MFC) at $0.6-7.5,0.15-0.60$ and $0.3-2.5 \mathrm{mg} / \mathrm{mL}$. The most sensitive microfungi were Trichoderma viride and Aspergillus versicolor, on the other hand, Aspergillus niger was the most resistant to the tested extracts. Commercial antifungal agents, bifonazole (MIC $0.10-0.20 \mathrm{mg} / \mathrm{mL}$; MFC $0.20-0.25 \mathrm{mg} / \mathrm{mL}$ ) and ketoconazole (MIC $0.15-2.50 \mathrm{mg} / \mathrm{mL}$; MFC $0.20-3.50 \mathrm{mg} / \mathrm{mL}$ ) were in general more active than the investigated extracts, with exception of Penicillium verrucosum and Penicillium funciculosum and ketoconazole. Ethyl acetate and methanol 80\% extracts showed higher antifungal effect toward $P$. verrucosum than ketoconazole. Furthermore, all the tested extracts showed higher fungicidal activity against $P$. funiculosm than ketoconazole. Ethyl acetate extract showed the highest antifungal potential, while dichloromethane extract showed the lowest antifungal effect.

\subsection{Cytotocix activity}

The three extracts proved cytotoxic effect against different human tumor cell lines (Table 4). Dichloromethane extract was the most active against all the tested tumor cell lines, HCT15 (colon carcinoma; $\mathrm{GI}_{50} 58.43 \mu \mathrm{g} / \mathrm{mL}$ ), HepG2 (hepatocellular carcinoma, $\mathrm{GI}_{50} 86.68 \mu \mathrm{g} / \mathrm{mL}$ ), HeLa (cervical carcinoma, $\mathrm{GI}_{50}$ $59.52 \mu \mathrm{g} / \mathrm{mL}$ ), MCF-7 (breast carcinoma, $\mathrm{GI}_{50} 72.84 \mu \mathrm{g} / \mathrm{mL}$ ), and $\mathrm{NCI}-\mathrm{H} 460$ (lung carcinoma; $\mathrm{GI}_{50} 74.15 \mu \mathrm{g} / \mathrm{mL}$ ). Nevertheless, this extract and methanol $80 \%$ extract showed toxicity to non-tumor cells (PLP2) at $190.71 \mu \mathrm{g} / \mathrm{mL}$ and $296.53 \mu \mathrm{g} / \mathrm{mL}$, respectively. It should be highlighted that the toxic concentrations for non-tumor cells are higher than the ones observed for tumor cell lines. The ethyl acetate extract showed the lowest anti-tumor potential $\left(\mathrm{GI}_{50}\right.$ values between 88.99 and $295.53 \mu \mathrm{g} / \mathrm{mL}$ ), but it did not show any 
Table 2

Antibacterial activity of the extracts from Alnus rugosa aerial parts.

\begin{tabular}{|c|c|c|c|c|c|}
\hline Bacteria & Dichloromethane MIC MBC (mg/mL) & Ethyl acetate MIC MBC (mg/mL) & Methanol 80\% MIC MBC (mg/mL) & Streptomycin MIC MBC (mg/mL) & Ampicillin MIC MBC (mg/mL) \\
\hline Bacillus cereus & $0.3 \pm 0.030 .4 \pm 0.03$ & $0.3 \pm 0.020 .6 \pm 0.02$ & $0.4 \pm 0.010 .5 \pm 0.00$ & $0.05 \pm 0.0030 .1 \pm 0.02$ & $0.1 \pm 0.020 .15 \pm 0.02$ \\
\hline Micrococcus flavus & $1.0 \pm 0.032 .0 \pm 0.20$ & $0.5 \pm 0.000 .6 \pm 0.06$ & $0.5 \pm 0.020 .6 \pm 0.02$ & $0.125 \pm 0.0030 .25 \pm 0.02$ & $0.1 \pm 0.0070 .15 \pm 0.00$ \\
\hline Staphylococcus aureus & $0.6 \pm 0.031 .0 \pm 0.07$ & $0.3 \pm 0.0070 .4 \pm 0.03$ & $0.4 \pm 0.020 .5 \pm 0.00$ & $0.25 \pm 0.00 .5 \pm 0.03$ & $0.1 \pm 0.010 .15 \pm 0.01$ \\
\hline Listeria monocytogenes & $2.0 \pm 0.14 .0 \pm 0.03$ & $2.5 \pm 0.025 .0 \pm 0.2$ & $1.25 \pm 0.032 .5 \pm 0.1$ & $0.15 \pm 0.0070 .3 \pm 0.02$ & $0.15 \pm 0.020 .30 \pm 0.00$ \\
\hline Escherichia coli & $1.0 \pm 0.072 .0 \pm 0.2$ & $0.6 \pm 0.021 .25 \pm 0.08$ & $0.6 \pm 0.002 .5 \pm 0.06$ & $0.50 \pm 0.020 .1 \pm 0.00$ & $0.30 \pm 0.030 .50 \pm 0.07$ \\
\hline Pseudomonas aeruginosa & $0.3 \pm 0.020 .5 \pm 0.02$ & $0.6 \pm 0.021 .25 \pm 0.02$ & $0.3 \pm 0.030 .5 \pm 0.00$ & $0.05 \pm 0.000 .1 \pm 0.003$ & $0.10 \pm 0.0030 .2 \pm 0.007$ \\
\hline Enterobacter cloacae & $1.0 \pm 0.002 .0 \pm 0.07$ & $0.4 \pm 0.030 .5 \pm 0.00$ & $0.4 \pm 0.020 .5 \pm 0.03$ & $0.05 \pm 0.0040 .1 \pm 0.02$ & $0.15 \pm 0.0060 .20 \pm 0.02$ \\
\hline Salmonella typhimurium & $1.0 \pm 0.102 .0 \pm 0.007$ & $1.25 \pm 0.082 .5 \pm 0.2$ & $1.25 \pm 0.082 .5 \pm 0.03$ & $0.05 \pm 0.000 .10 \pm 0.00$ & $0.15 \pm 0.010 .20 \pm 0.03$ \\
\hline
\end{tabular}

MIC-minimum inhibitory concentration; MFC-minimum bactericidal concentration.

$.082 .5 \pm 0.03$

Table 3

Antifungal activity of the extracts from Alnus rugosa aerial parts.

\begin{tabular}{|c|c|c|c|c|c|}
\hline Fungi & Dichloromethane MIC MFC (mg/mL) & Ethyl acetate MIC MFC $(\mathrm{mg} / \mathrm{mL})$ & Methanol 80\% MIC MFC (mg/mL) & Bifonazole MIC MFC $(\mathrm{mg} / \mathrm{mL})$ & Ketoconazole MIC MFC (mg/mL) \\
\hline Aspergillus fumigatus & $2.5 \pm 0.205 .0 \pm 0.07$ & $0.3 \pm 0.030 .6 \pm 0.03$ & $1.25 \pm 0.082 .5 \pm 0.03$ & $0.15 \pm 0.000 .20 \pm 0.02$ & $0.20 \pm 0.030 .50 \pm 0.00$ \\
\hline Aspergillus versicolor & $0.3 \pm 0.020 .6 \pm 0.03$ & $0.3 \pm 0.030 .6 \pm 0.00$ & $0.3 \pm 0.000 .6 \pm 0.02$ & $0.10 \pm 0.0070 .20 \pm 0.008$ & $0.20 \pm 0.020 .50 \pm 0.02$ \\
\hline Aspergillus ochraceus & $1.2 \pm 0.15 .0 \pm 0.3$ & $0.2 \pm 0.030 .6 \pm 0.03$ & $0.2 \pm 0.020 .6 \pm 0.02$ & $0.15 \pm 0.020 .20 \pm 0.00$ & $0.15 \pm 0.000 .20 \pm 0.03$ \\
\hline Aspergillus niger & $2.5 \pm 0.207 .5 \pm 0.20$ & $0.2 \pm 0.020 .6 \pm 0.00$ & $0.2 \pm 0.020 .6 \pm 0.03$ & $0.15 \pm 0.010 .20 \pm 0.02$ & $0.20 \pm 0.030 .50 \pm 0.03$ \\
\hline Penicillium verrucosum & $1.25 \pm 0.105 .0 \pm 0.07$ & $0.15 \pm 0.0070 .3 \pm 0.01$ & $0.2 \pm 0.0070 .6 \pm 0.02$ & $0.15 \pm 0.000 .20 \pm 0.02$ & $1.0 \pm 0.071 .0 \pm 0.00$ \\
\hline Penicillium ochrochloron & $0.6 \pm 0.03^{\mathrm{a}} 2.5 \pm 0.00^{\mathrm{b}}$ & $0.15 \pm 0.000 .3 \pm 0.02$ & $0.3 \pm 0.030 .6 \pm 0.03$ & $0.20 \pm 0.020 .25 \pm 0.01$ & $0.20 \pm 0.020 .50 \pm 0.02$ \\
\hline Penicillium funiculosum & $0.3 \pm 0.00^{c} 0.6 \pm 0.03^{d}$ & $0.075 \pm 0.0080 .15 \pm 0.007$ & $0.15 \pm 0.0070 .3 \pm 0.00$ & $0.20 \pm 0.020 .25 \pm 0.02$ & $2.5 \pm 0.203 .5 \pm 0.10$ \\
\hline Trichoderma viride & $0.3 \pm 0.02^{\mathrm{e}} 0.6 \pm 0.02^{\mathrm{e}}$ & $0.3 \pm 0.000 .6 \pm 0.02$ & $0.3 \pm 0.030 .6 \pm 0.00$ & $0.10 \pm 0.0070 .20 \pm 0.03$ & $0.20 \pm 0.030 .30 \pm 0.03$ \\
\hline
\end{tabular}

MIC-minimum inhibitory concentration; MFC-minimum fungicidal concentration. 
Table 4

Cytotoxicity of different extracts from Alnus rugosa in human tumor cell lines and in non-tumor liver primary culture.

\begin{tabular}{|c|c|c|c|c|}
\hline Cell line & Dichloromethane $\mathrm{GI}_{50}$ values $(\mu \mathrm{g} / \mathrm{mL})$ & Ethyl acetate $\mathrm{GI}_{50}$ values $(\mu \mathrm{g} / \mathrm{mL})$ & Methanol $80 \% \mathrm{GI}_{50}$ values $(\mu \mathrm{g} / \mathrm{mL})$ & Elipticine $\mathrm{GI}_{50}$ values $(\mu \mathrm{g} / \mathrm{mL})$ \\
\hline HCT15 & $58.43 \pm 3.88$ & $88.99 \pm 5.92$ & $73.96 \pm 6.84$ & $0.91 \pm 0.04$ \\
\hline HepG2 & $86.68 \pm 5.90$ & $295.53 \pm 18.53$ & $276.66 \pm 2.63$ & $1.42 \pm 0.00$ \\
\hline HeLa & $59.52 \pm 4.74$ & $167.19 \pm 13.97$ & $69.73 \pm 1.55$ & $1.91 \pm 0.06$ \\
\hline MCF7 & $72.84 \pm 4.76$ & $264.78 \pm 22.34$ & $241.38 \pm 4.95$ & $1.14 \pm 0.21$ \\
\hline $\mathrm{NCI}-\mathrm{H} 460$ & $74.15 \pm 2.87$ & $264.98 \pm 5.68$ & $199.88 \pm 7.42$ & $3.22 \pm 0.67$ \\
\hline PLP2 & $190.71 \pm 3.85$ & $>400$ & $296.53 \pm 10.93$ & $2.06 \pm 0.03$ \\
\hline
\end{tabular}

toxicity for normal liver cells $\left(\mathrm{GI}_{50}>400 \mu \mathrm{g} / \mathrm{mL}\right.$ ). The most susceptible tumor cell line for dichloromethane and ethyl acetate extracts was HCT-15, while HeLa cell line was the most susceptible for methanol $80 \%$ extract.

\section{Discussion}

The plants of Alnus genus contained various types of plant secondary metabolites including terpenoids, flavonoids, diarylheptanoids, phenols, steroids, tannins, and many others (Sati et al., 2011). Martineau et al. (2010) reported the results of a classical bioassay-guided fractionation approach aimed at identifying the active principles responsible for the inhibition of adipogenesis, as measured using triglyceride accumulation in the 3T3-L1 adipocyte model cell line. The glycosides oregonin and salicortin were isolated and identified as the respective active principles for Alnus incana ssp. rugosa. These compounds thus offer promise as novel agents to mitigate the incidence or the progression of obesity. The major bioactive components identified in our work from $A$. rugosa dichloromethane extract were $\beta$-sitosterol, $\beta$-sitosterol 3-O- $\beta$-glucoside, and apigenin, while from ethyl acetate extract, diosmetin, narigenin, luteolin, and luteolin 7-O- $\beta$-glucoside were the most abundant. Quercetin 3-O$\alpha$-rhamnoside, isorhamnetin 3-O- $\beta$-glucoside, and isorhamnetin $3-O$ - $\beta$-glucoside-7- $O-\alpha$-rhamnoside were identified in methanol $80 \%$ extract. The chemical investigation of Alnus species has revealed many components from this genus with significant bioactivities. On the basis of the above discussion, it may be concluded that all the members of genus Alnus have different biological activities. The genus is well known for its traditional medicinal uses including cancer, hepatitis, inflammation of uterus, uterine cancer, rheumatism, dysentery, stomachache, diarrhea, and fever, etc. The plants contain variety of bioactive constituents, i.e., triterpenoids, flavonoids, tannins, phenols, steroids, diarylheptanoids, etc. Among them, diarylheptanoids (hirsutenone, oregonin, (5S)-O-methylhirsutanonol, 2-oxatrycyclo[13.2.2.13,7]eicosa3,5,7(20),15,17,18-hexaen-10-16-diol, 2-oxatrycyclo [13.2.2.13,7] eicosa-3,5,7-(20),15,17,18-hexaen-10-one,

garugamblin-3, acerogenin L, etc.) are highly potent bioactivities (Sati et al., 2011). Middleton et al. (2005) tested antibacterial activity of $A$. glutinosa. $\mathrm{MeOH}$ extract was found to be active against the following eight bacterial species: Citrobacter freundii, E. coli, Klebsiella aerogenes, Lactobacillus plantarum, P. aeruginosa, S. aureus, and the most potent activity was against $E$. coli with an MIC value of $1.25 \times 10^{-1} \mathrm{mg} / \mathrm{mL}$. Antimicrobial activity of methanol extract of $A$. rubra bark against gram-positive and gram-negative bacteria. Diarylheptanoid and its glycoside (oregonin) were identified as the two constituents responsible for this activity. A. rugosa contained tannin with a broad-spectrum of antibiotic activity, but there is no evidence that the extracts or compounds from $A$. rugosa were tested. Its close relation, A. rubra, which was often used in its stead in the last century, has demonstrated an antibiotic effect against both fungal and bacterial infection. It even demonstrated antibiotic activity against methicillin resistant $S$. aureus (Saxena et al., 1995). This species and its antimicrobial activity should be more examined. Ethyl acetate extract of $A$. rugosa tested in our work showed the highest antifungal potential, while dichloromethane extract showed the lowest antifungal effect.

Choi et al. (2008) analyzed the cytotoxic activity of isolated from Alnus japonica bark against murine B16 melanoma, human SNU-1 gastric cancer, human SNU-354 hepatoma cancer, and human SNU-C4 colorectal tumor cell lines. The diarylheptanoids showed potent cytotoxic activity against murine B16 melanoma cells and human SNU-C1 gastric cancer cell when the cell viability was analyzed by MTT (3-(4,5-dimethylthiazol-2-yl)2,5-diphenyltetrazoliumbromide) assay. There is no available data for cytotoxic activity of the extracts or compounds from A. rugosa. In this present research, dichloromethane extract was the most active against all the tested tumor cell lines namely, HCT-15 (colon carcinoma; $\mathrm{GI}_{50} 58.43 \mu \mathrm{g} / \mathrm{mL}$ ), HepG2 (hepatocellular carcinoma, $\mathrm{GI}_{50} 86.68 \mu \mathrm{g} / \mathrm{mL}$ ), HeLa (cervical carcinoma, GI $59.52 \mu \mathrm{g} / \mathrm{mL}$ ), MCF-7 (breast carcinoma, $\mathrm{GI}_{50} 72.84 \mu \mathrm{g} / \mathrm{mL}$ ) and NCI-H460 (lung carcinoma; $\mathrm{GI}_{50} 74.15 \mu \mathrm{g} / \mathrm{mL}$ ).

\section{Conclusion}

The extracts of A. rugosa aerial parts were found to be promising antibacterial and antifungal agents against human pathogens and also as anticancer agents. Different compounds were identified in the extracts that could be responsible for the observed biological activity, namely $\beta$-sitosterol, $\beta$-sitosterol $3-O-\beta$-glucoside and apigenin of dichloromethane extract; diosmetin, naringenin, luteolin, and luteolin 7-O- $\beta$-glucoside of ethyl acetate extract, and quercetin 3-O- $\alpha$-rhamnoside, isorhamnetin $3-O-\beta$-glucoside and isorhamnetin 3- $\beta$ - $O$-glucoside-7-O- $\alpha$-rhamnoside of methanol $80 \%$ extract. Complete chemical screening of $A$. rugosa is necessary. At the moment, there is only a preliminary data of the species chemical constituents and their bioactivity. Results obtained in this paper gives contribution that this plant is a potential source of valuable compounds with a broad spectrum of activity.

\section{Conflict of interest statement}

There is no conflict of interest associated with the authors of this paper.

\section{Acknowledgments}

The authors are grateful to strategic project PEstOE/AGR/UI0690/2011 for financial support to CIMO. R. Calhelha thanks to FCT, POPH-QREN and FSE for his grant (SFRH/BD/78307/2011), and Serbian Ministry of Education, Science and Technological Development, grant number 173032 for financial support.

\section{References}

Choi, S.E., Kim, K.H., Kwon, J.H., Kim, S.B., Kim, H.W., Lee, M.W., 2008. Cytotoxic activity of diarylheptanoids from Alnus japonica. Arch. Pharm. Res. 31, 1287-1289. CLSI (Clinical and Laboratory Standards Institute), 2009. Methods for dilution antimicrobial susceptibility tests for bacteria that grow aerobically, Approved standard. In: CLSI Publication M07-A8, eighth ed. Clinical and Laboratory Standards Institute, Wayne, PA. 
Connolly, J.D., Overton, K.H., Polonsky, J., 1970. The chemistry and biochemistry of the linonoids and quassinoids. In: Reinhold, L., Liwashitz, Y. (Eds.), Programme Phytochemistry. Wiley, London, p. 385.

Dimayuga, R.E., Garcia, S.K., 1991. Antimicrobial screening of medicinal plants from Baja California sur, Mexico. J. Ethnopharmacol. 31, 181-192.

Ersoz, T., Harput, U.S., Saracolglu, U., Calis, T., 2002. Phenolic compounds from Scutellaria pontica. Turk. J. Chem. 26, 581-588.

Espinel-Ingroff, A., 2001. Comparation of the E-test with the NCCLS M38-P method for antifungal susceptibility testing of common and emerging pathogenic filamentous fungi. J. Clin. Microbiol. 39, 1360-1367.

Farnsworth, N.R., 1966. Biological and phytochemical screening of plants. J. Phram. Sci. 55, 225-276.

Geissman, T.A., 1962. The Chemistry of Flavonoids Compounds. Pergamon, London, pp. 126-129.

Gennari, C.D., Castoldi, B., Sharon, O., 2007. Natural products with taxol-like antitumor activity: synthetic approaches to eleutherobin and dictyostatin. Pure Med. Appl. Chem. 79, 173-180

Guimarães, R., Barros, L., Dueñas, M., Calhelha, R.C., Carvalho, A.M., Santos-Buelga, C., Queiroz, M.J.R.P., Ferreira, I.C.F.R., 2013. Nutrients, phytochemicals and bioactivity of wild Roman chamomile: a comparison between the herb and its preparations. Food Chem. 136, 718-725.

Hanel, H., Raether, W., 1988. A more sophisticated method of determining the fungicidal effect of water-insoluble preparations with a cell harvester, using miconazole as an example. Mycoses 31, 148-154.

Harborne, J.B., 1973. Phytochemicalmethods. Chapman \& Hall, London.

Huxley, A., 1992. The New RHS Dictionary of Gardening.

Levy, S.B., Marshall, B., 2004. Antibacterial resistance worldwide: causes, challenges and responses. Nat. Med. 10, 122-129.

Martineau, L.C., Muhammad, A., Saleem, A., Hervé, J., Harris, C., Arnason, J.T., Haddad, P.S., 2010. Anti-adipogenic activities of Alnus incana and Populus balsamifera bark extracts, Part II: Bioassay-guided Identification of actives salicortin and oregonin. Planta Med. 76, 1519-1524.

Middleton, P., Stewart, F., Al-Qahtani, S., Egan, P., Rourke, C., Abdulrahman, A., 2005. Antioxidant, antibacterial activities and general toxicity of Alnus glutinosa, Fraxinus excelsior and Papaver rhoeas. Iran. J. Pharm. Res. 2, 81-86.
Morris, A.K., Masterton, R.G., 2002. Antibiotic resistance surveillance: action for international studies. J. Antimicrob. Chemother. 49, 7-10.

Parekh, J., Chanda, V., 2007. In vitro antimicrobial activity and phytochemical analysis of some Indial medicinal plants. Turk. J. Biol. 31, 53-58.

Pateh, U.U., Haruna, A.K., Garba, M., Iliya, I.M., Abubakar, M.S., Ambi, A.A., 2009 Isolation of stigmasterol, $\beta$-sitosterol and 2-hydroxyhexadecanoic acid methy ester from the rhizomes of Stylochiton lancifolius and Kotchy (Araceae). Niger. J. Pharm. Sci. 7, 19-25.

Sati, O.P., Sati, N., Sati, S.C., 2011. Bioactive constituents and medicinal importance of genus Alnus. Pharamacol. Rev. 5, 174-183.

Saeidnia, S., Nourbakhsh, M.S., Gohari, A.R., Davood, A., 2011. Isolation and identification of the main compounds of Satureja sahendica Bornm. Aust. J. Basic Appl. Sci. 5, 1450-1453.

Saxena, G., Farmer, S., Hancok, R.E., Towers, G.H., 1995. Antimicrobial compounds from Alnus rubra. Int. J. Pharmacogn. 33, 33-36.

Saxena, k., Goutam, A., 2008. Isolation and study of the flavone glycoside; Luteolin7-O- $\beta$-D-glucopyranoside from the seeds of the Capparis decidua (FORSK). Int. J. Chem. Sci. 6, 7-10.

Soliman, F.M., Shehata, A.H., Khaleel, A.E., Ezzat, S.M., 2002. An acylated kaempferol Glycoside from flowers of Foeniculum vulgare and F. dulce. Molecules 7, 245-251.

Tsukatani, T., Suenaga, H., Shiga, M., Noguchi, K., Ishiyama, M., Ezoe, T., Matsumoto K., 2012. Comparison of the WST-8 colorimetric method and the CLSI broth microdilution method for susceptibility testing against drug-resistant bacteria. J. Microb. Met. 90, 160-166.

Wang, D.M., Wen-Jun, P., Yong-Hong, W., Yu-Juan, Z., Shan-Shan, W., 2012. A new isorhamnetin glycoside and other phenolic compounds from Callianthemum taipaicum. Molecules 17, 4595-4603.

Wolf, H.H., Swinyard, E.A., Goodman, L.S., 1962. Anticonvulsant properties of some N-substituted hydanoins. J. Phram. Sci. 51, 74-76.

Yinhong, W., Daowan, L., Yongmin, Z., Along, K., Yongxiao, C., Wenji, S., 2009. Study of steroidal saponins in Dioscorea zingiberensis C.H. Wright. J. Nat. Prod. 2 123-132. 